\title{
Acercamiento a un estudio sobre toma de posiciones y disposiciones en la organización. El caso de una empresa desde la perspectiva Bourdiana
}

Approach to a Study on Position Taking and Dispositions within the Organization; a Company Case from the Bourdian Perspective

\author{
Approche a une étude sur la prise de positions et de dispositions dans une organisation. Le cas d'une \\ entreprise à partir de la perspective bourdieusienne
}

\author{
Daimer Higuita López \\ Profesor, Facultad de Ciencias Económicas, Universidad \\ Nacional de Colombia, Bogotá, Colombia. \\ Administrador de empresas, Universidad de Antioquia, \\ Colombia, PhD en Ciencias Económicas, Universidad Nacional \\ de Colombia, Colombia. \\ E-mail:dhiguital@unal.edu.co
}

Artículo de investigación científica y tecnológica Según clasificación COLCIENCIAS

Recibido: enero-5-15 Revisado: marzo-30-15 Aprobado: abril-23-15

\section{Resumen}

El estudio que dio pie a este documento se desarrolló a partir de distintas medidas implementadas por la gerencia de una organización que buscaba transformar las formas de trabajar y las maneras de relacionarse. El propósito es exponer que las posiciones y la toma de posiciones de los trabajadores fueron factores importantes en la transformación de la cultura de esta organización. Y para ello, la exposición se apoya en los postulados de Bourdieu $(1995,2000)$ y de Emirbayer y Johnson (2008). El estudio produjo datos empíricos con trabajadores y directivos a través de entrevistas, grupos focales, encuestas, procesos de observación y el análisis de documentos. De esta forma, el texto expone: a) cómo se fomentó la transformación de la cultura, b) la aprehensión de nuevos rasgos culturales y la participación del habitus en este proceso, c) las acciones que emprenden los trabajadores y los intercambios simbólicos con la organización, d) las tensiones rastreadas y las agrupaciones que se dieron entre los trabajadores, producto de la toma de posición de estos, lo que sugiere que en ese proceso de transformación cultural y la toma de posiciones de los trabajadores ha jugado un rol fundamental.

Palabras clave: campo, habitus, transformación cultural, toma de posición.

\section{Abstract}

The study that led to this document was developed from different measures implemented by management in an organization, which sought to transform ways of working and of relating. The purpose was to expose that the positions and the taking of positions by workers were important factors in the transformation in this organization's culture. For this, the exposition is supported by the postulates of Bourdieu $(1995,2000)$ and by Emirbayer and Johnson (2008). The study produced empirical data with workers and directors through interviews, focal groups, surveys, observation processes, and analysis of documents. Thus, the text exposes: a) how the culture transformation was promoted, b) apprehension of new cultural traits and the participation of the habitus in this process, c) actions undertaken by workers and the symbolic exchange with the organization, d) tensions traced and the groups created among workers, product of their position taking, which suggests that within this cultural transformation process and the taking of positions the workers have played a fundamental role.

\section{Résumée}

Keywords: filed, habitus, cultural transformation, position taking.

L’étude qui a permis l`idée de ce document s’est développée à partir de différentes mesures appliquées par la gérance d’une organisation qui cherchait à transformer les façons de travailler et les modes de relation. L’objectif est de mettre en évidence que les positions et la prise de position des travailleurs ont été d’importants facteurs pour la transformation de la culture de cette organisation. Pour ceci, l’étude s’appuie sur les postulats de Bourdieu (1995,2000) et d’ Emirbayer et Johnson (2008); et a produit des données empiriques avec les travailleurs et les directeurs par le biais d’interviews, groupes de discussion, enquêtes, processus d’observation et analyse de documents, de cette façon, le texte expose : a) la façon comme s'est inciter la transformation de la culture; b) l’appréhension de nouvelles caractéristiques culturelles et la participation de l' habitus dans ce processus ; c) les actions entreprises par les travailleurs et les échanges symboliques avec l'organisation; d) les tensions suivies et les groupes conformés parmi les travailleurs, produit de leur prise de position, ce qui suggère que dans ce processus de transformation culturelle la prise de position des travailleurs joue un rôle fondamental.. 


\section{Introducción}

En el año 2003, llega un nuevo gerente general a la organización objeto de estudio, la cual hace parte del sector energético en Colombia. A partir del 2005, tomando como referencia a Semco ${ }^{1}$, el gerente de la empresa del sector energético se propuso implementar nuevas maneras de comportarse, de trabajar y de organizarse, para promover la filosofía "calidad a tiempo". Se propuso transformar la cultura. Hoy, los trabajadores y participantes del estudio hablan de una modificación cultural. Se sabe que desde una perspectiva funcionalista la organización tiene una cultura (Thevenet, 2013) y lo que se busca es gestionar o crear pieza por pieza esa cultura. Se sabe también, que desde una mirada interpretativa la organización es una cultura (Smircich, 1983; Aktouf, 2002; Muñoz, 2010), y lo que se intenta es comprender su proceso de configuración. Por tanto, el propósito de este documento es analizar cómo las posiciones y la toma de posiciones de los trabajadores participaron en esta transformación.

En esa medida, el texto se ha organizado en cinco partes. En primer lugar, se presenta la revisión de la literatura. Después se exponen algunos elementos del habitus y el campo, apoyados en Bourdieu $(1995,2000)$ $y$ en Emirbayer y Johnson (2008). El abordaje de estas categorías será fundamental para comprender la toma de posiciones de los sujetos. Luego se delinea la metodología empleada en el estudio de caso. En la cuarta parte se exponen y discuten hallazgos. Allí se indica brevemente cómo se fomentó la transformación de la cultura en esa organización; se aborda la aprehensión de nuevos rasgos culturales y la participación en este proceso del habitus; se exponen las acciones que emprenden los trabajadores (es decir, la toma de posiciones), los bienes sociales que se construyen en el colectivo y los intercambios simbólicos; se dibujan las tensiones rastreadas en esta organización y las agrupaciones que se fueron dando entre los trabajadores, producto de la toma de posición de ellos. Finalmente, el documento termina con algunas conclusiones.

\section{Revisión de la literatura}

Ya son varios los trabajos que traen categorías como habitus, campo y otras propuestas de Pierre Bourdieu, al terreno organizacional. Strategor en 1995 planteó que los trabajadores llegan a la organización con un habitus, el cual les sirve de brújula para sus acciones². Más recientemente, Emirbayer y Johnson (2008) exponen por qué la organización puede ser abordada como si fuera un campo. Ahora bien, en el caso particular del uso de categorías bourdiesianas a la cultura, el trabajo de Ojeda (2009) es un ejemplo de la aplicación de los habitus a la cultura organizacional, en la que este autor ve a los individuos dotados de una historia.

Adicionalmente, Hallett (2003), a partir del paralelo entre las propuestas de Bourdieu y Goffman propone el orden negociado entre los distintos actores (o trabajadores) como una forma de configurar la cultura. De otro lado, el reconocido crítico Alvesson (1994, 2008) articula las improntas individuales a las lógicas de la identidad en las organizaciones. Dalton (2004) sugiere que la creatividad necesita una adaptación de los hábitos a contextos específicos de acción, dependiendo del habitus. Lam y Robertson (2010) sostienen que la cultura y el contexto en la empresa tienen influencia en disposiciones como la de participar. Sin embargo, Bordia, Hobman, Jones, Gallois, y Callan (2004) argumentan que la participación en decisiones está asociada con un sentimiento de control en los trabajadores. Gartman (2007) y Mangi (2009), desde la sociología cultural y desde el neoinstitucionalismo, también hacen aportes que se pueden trasladar a las organizaciones. McDonough y Polzer (2012) rastrean la noción de histéresis en el cambio organizacional en el sector público y Sieweke (2014) revisa la aplicación de la teoría de Pierre Bourdieu a los denominados management and organization studies (MOS).

\section{Del habitus, el campo y la toma de posición}

Antes de explicar en qué consiste el tema de estudio, es necesario plantear una definición sobre la noción de cultura y proponer algunas reflexiones sobre la transformación cultural. La cultura se entendió en la investigación como un "conjunto ligado de maneras de pensar, de sentir y de actuar que, siendo aprendidas y comprendidas por una pluralidad de personas, sirven para construir a esas personas en una colectividad particular diferenciada" (Aktouf, 2002, p. 23). Además, la cultura organizacional se entendió como un esquema de referencia para patrones de información, comportamiento y actitudes que compartimos con otros en el trabajo (...) que se enriquece con los aportes de los individuos, se perpetúa a través de ellos, a la vez que las personas enriquecen sus otros entornos (Zapata y Rodríguez, 2008, p. 136).

Como se dijo, el gerente de la organización objeto de estudio se propuso implementar nuevas maneras de comportarse, de trabajar y de organizarse. Ahora bien, si los trabajadores sostienen que hoy hay efectivamente nuevas prácticas en este espacio social, se consideró importante analizar en esta organización, las posiciones de los trabajadores y la toma de posiciones de los trabajadores en el proceso de transformación cultural. Y para ello, la noción de habitus (entendida como disposiciones)

${ }^{1}$ Semco es la empresa brasilera de metalmecánica que se hizo exitosa a nivel mundial y que, entre otras medidas, eliminó la estructura organizacional jerárquica, propició la participación de sus trabajadores y dio mayor flexibilidad en las formas de trabajar. 
ofrece una comprensión alternativa (más no la única) sobre las acciones de las personas, porque presenta diferentes racionalidades prácticas de los sujetos, que son función de sus esquemas de acción y percepción formados en unas condiciones materiales particulares.

Por habitus Bourdieu (2002) entiende el conjunto de esquemas generativos a partir de los cuales los sujetos perciben el mundo y actúan en él. Estos esquemas han sido conformados a lo largo de la historia de cada sujeto y se constituyen en estructuras estructurantes. Bourdieu y Wacquant (1995) presentan la génesis del habitus como proceso de inculcación de un arbitrario cultural y como incorporación de determinadas condiciones de existencia, lo cual plantea dos modos distintos de generación del habitus: la inculcación y la incorporación. La inculcación supone una acción pedagógica efectuada dentro de un espacio institucional (familiar o escolar, y organizacional, como bien lo afirma Sainsaulieu, 1997) por actores dotados de autoridad de delegación, que imponen normas valiéndose de técnicas disciplinarias.

La incorporación, en cambio, remite a la idea de una interiorización por los sujetos de las regularidades inscritas en sus condiciones de existencia. Y esta interiorización sugiere un aprendizaje cultural. En este sentido, la cultura lleva consigo la interiorización de cada situación hecha por el individuo. $Y$ a tal estado de cultura, afirma Aktouf (2002), no se llega sino en la medida en que los miembros perciben una identidad común.

Considerar el concepto de habitus para estudiar la cultura en la organización, nos lleva la noción de campo. Para Bourdieu, el habitus no tiene valor sino en un espacio social (2007, p. 208). El campo está concebido como "un sistema de líneas de fuerza en el que los agentes que forman parte de él pueden ser descritos como otras tantas fuerzas que, posicionándose, oponiéndose y componiendo, le confieren su estructura especifica en un momento dado del tiempo" (Bourdieu, 2007, p. 191).

Ahora bien, la organización vista como un campo está conformada por estructuras, interacciones y por diversos actores. Cada uno de esos actores tienen unas posiciones y toman posiciones, configurando las fuerzas del campo. Emirbayer y Johnson (2008) ven el campo organizacional desde dos ángulos: las posiciones objetivas de los actores y su toma de posiciones. Para ellos, esto se corresponde con los conceptos de relaciones sociales y de cultura, respectivamente. Ahora bien, esas posiciones estarían mediadas por las disposiciones (o habitus) que tiene el trabajador producto de su historia incorporada. Por consiguiente, Emirbayer y Johnson (2008), sostienen que la organización puede ser abordada como un campo, en el que estas dos situaciones (posiciones y toma de posiciones) se constituyen mutuamente. Y este "juego" de posiciones y toma de posiciones de los trabajadores parece participar en la transformación de la cultura y ese es el propósito del presente texto. En otras palabras, interesa ver cómo los trabajadores van tomando posiciones dentro de ese espacio social denominado organización y cómo se va dando la incorporación de rasgos culturales. Como veremos en el apartado hallazgos, esa toma de posición se traduce en acciones concretas ejecutadas individual o grupalmente por los trabajadores.

Analizar las posiciones y la toma de posiciones de los trabajadores, como factores clave en la transformación de la cultura (objeto del presente manuscrito), requiere considerar dos conceptos claves presentes en el campo. Bourdieu (2002) manifiesta que en determinadas situaciones se puede producir el fenómeno de "histéresis de habitus", donde las disposiciones aparecen mal ajustadas a las condiciones presentes porque están objetivamente ajustadas a otro tipo de condiciones. Por ejemplo, una persona que se formó en "ambientes" de cooperación (su familia, la universidad, otras organizaciones) y luego ingresa a una organización con un ambiente altamente competitivo entre sus miembros, podría vivir este tipo de circunstancias en la transformación de la cultura. En contraste, cuando el habitus entra en contacto con un mundo social del cual es producto, se encuentra como "pez en el agua" y el entorno le parece autoevidente (Bourdieu y Wacquant, 1995, p. 88). Bourdieu (1995, p. 88) propone que cuando el habitus enfrenta condiciones semejantes a aquellas de las cuales es producto, está perfectamente adaptado a ellas sin necesidad de hacer ningún esfuerzo de adaptación intencional. En el caso de la organización en estudio, cuando una manifestación cultural particular (como un valor corporativo, por ejemplo) esté más cerca de las disposiciones del empleado y sus propios valores, esta manifestación podría serle, en algún sentido, más familiar. De allí que al analizar la transformación cultural en la organización objeto de estudio, se haya puesto la mirada en las posiciones y en la toma de posiciones de los trabajadores. Veamos entonces la metodología seguida en el estudio.

\section{Metodología}

La producción de información durante la investigación tuvo dos etapas. En la primera, se buscaba identificar qué tenían en mente los directivos en términos de gestión cultural, es decir, qué deseaban transformar y cómo buscaban hacerlo ${ }^{3}$. En esa etapa se hicieron entrevistas semiestructuradas a directivos y se analizaron documentos escritos. En la segunda, se indagó la importancia que los trabajadores le asignan a las medidas implementadas. Asimismo, se exploraron qué hábitos se fueron formando a partir de esas medidas y las posiciones y la toma de posiciones de los empleados. Para ello se efectuaron grupos foco y entrevistas en profundidad con trabajadores. Cada una de estas técnicas buscaba validar la información que se había producido con las anteriores. Adicional y simultáneamente, se realizaron siete visitas de observación en la organización. A continuación se detalla la aplicación de estas técnicas. 
Algunos de los interrogantes de las entrevistas apuntaban a indagar por el diseño de manifestaciones culturales, por los mecanismos de gestión cultural, por la filosofía y la historia de la compañía. En el caso de los ingenieros y de las personas del área de talento entrevistadas, estas fueron seleccionadas por el rol que han tenido en la configuración de mecanismos culturales (como grupos de discusión, etc.), por la información que poseían y por la disponibilidad para participar en el estudio. Estas entrevistas se desarrollaron siguiendo los criterios de saturación planteados por Galeano (2004) según los cuales cuando un nuevo participante no aporta nueva información, se revisa la que se ha producido hasta el momento.

Por el tamaño de la organización, se consideró que la encuesta era un instrumento adecuado para obtener gran cantidad de información relacionada con rasgos culturales (ver Tabla 2). La encuesta incluyó tanto preguntas abiertas como cerradas y tenía como propósito identificar: 1) cuáles son las manifestaciones culturales, las prácticas y los mecanismos de gestión cultural más recordados por los trabajadores; 2) cuáles eran las posiciones de los trabajadores; 3) hábitos asociados con valores, mecanismos, manifestaciones y filosofía organizacional. En total 199 personas respondieron la encuesta, la cual fue aplicada online. Como instrumento, la encuesta permitió rastrear variables sociodemográficas (útiles para el posterior análisis) así como disposiciones en los participantes.

Más que pretender generalizar los hallazgos, este estudio buscaba profundizar en una situación particular para rastrear las posiciones de los trabajadores y su toma de posiciones, en la transformación de la cultura de la organización objeto de estudio. Y para ahondar, era necesario utilizar distintas técnicas que permitieran ir validando la información producida. En este sentido, el grupo foco permitió que los mismos participantes pudieran profundizar en la información que se había recolectado con la encuesta. En total se realizaron nueve grupos foco. Cada grupo estuvo conformado en promedio por siete personas. Los propósitos del grupo foco era validar la información de las encuestas y entrevistas, indagar por la pertinencia que los trabajadores le ven a los mecanismos de gestión cultural y a las nuevas medidas implementadas, y rastrear la toma de posiciones. Recordar que en los estudios cualitativos "la muestra no se selecciona, se configura, es decir, se va estructurando a través de las diferentes etapas del proceso de recolección de datos" (Bonilla y Rodríguez, 2005, p. 134). Así que esta fue la dinámica que siguió tanto la aplicación de las entrevistas como la realización de los grupos foco.

Esta información se analizó a partir de la construcción de categorías siguiendo la metodología sugerida por Wodak y Meyer (2003), en la que, a partir del discurso de los informantes y su posterior validación con los mismos, se van configurando y llenando de contenido las categorías. En este caso, las categorías estaban asociadas a formas de trabajar y maneras de relacionarse que se derivan de las medidas propuestas por la gerencia (y que aparecen en la Tabla 1). ¿Por qué el grupo foco? Porque el propósito era validar que las personas en presencia de otras determinaran si habían aprehendido algún rasgo cultural que otros también habían aprehendido, pues siguiendo a Ember y Ember (1997), si una sola persona es la que hace "algo", estamos hablando de un hábito personal y no de un rasgo cultural.

En el caso de las entrevistas y los grupos foco, los participantes fueron seleccionados por el rol que han tenido en la configuración de mecanismos culturales (como el diseño de logos, etc.), por la información que poseían y por la disponibilidad para participar en el estudio. Es importante hacer las siguientes precisiones: 1) las técnicas se aplicaron en distintos momentos; 2) cada una buscaba validar la información que se venía recolectando y producir información adicional; 3) se analizó lo que cada una arrojaba tanto con la gerencia como a la luz de la teoría, antes de aplicar la siguiente; 4) luego, los resultados se volvieron a analizar en simultáneo. De allí que se indagara por las mismas categorías en los distintos momentos (encuesta, grupos foco y entrevistas).

Por su parte, la observación se aplicó en paralelo con el uso de las demás técnicas descritas acá. Observar implica focalizar la atención de manera intencional, sobre algunos segmentos de la realidad bajo estudio, con el fin de reconstruir inductivamente la dinámica de la situación (Bonilla y Rodríguez, 2005, p. 228). Se realizaron siete visitas a la organización con propósitos de observación, en las que se buscaba ir validando la información que arrojaban los demás instrumentos.

Para rastrear las posiciones y la toma de posiciones de los trabajadores, en el análisis se hicieron algunas contrastaciones. Como un paso previo a la categorización, se construyeron redes conceptuales (Alvesson y Karreman, 2011) a partir de los distintos elementos que emergieron de la interpretación de los datos, asociados con formas de trabajar y maneras de relacionarse que se derivan de las medidas propuestas por la gerencia. Por ejemplo, se tomaron los casos (encuestados) en los que el compromiso había recibido la calificación 1, en una escala de 1 a 13 (quienes le asignaban el valor 1 consideraban que el compromiso es lo que más identifica a un trabajador, entre trece factores). Esto se contrastó con otras preguntas para mirar cómo habían calificado esas mismas personas otros factores. Luego, este mismo rastreo se hizo para las personas que calificaron el compromiso con 2 y así sucesivamente, hasta los que lo calificaron con 13 (el último valor de la escala). 


\begin{tabular}{|l|l|}
\hline \multicolumn{2}{|c|}{ Tabla 1. Medidas y rasgos culturales deseados } \\
- Reducción de burocracia & • Enfocados en entrega al cliente, basados en laresponsabilidad \\
- Comunicación total, honesta y medida mensualmente & - Los empleados entrevistan sus posibles compañeros de trabajo \\
- Participación de empleados en decisiones & - Los empleados negocian con los clientes \\
- Aceptar que todos tratamos de hacer lo mejor & - No pagamos horas extras pero cada individuo decide los \\
- Basado en total confianza y respeto en la gente & - No hay procedimientos, hay guías \\
- 9o\% de los empleados con contratos a término indefinido. & - No hay bonos individuales/distribuido anualmente dependiendo \\
- No hay evaluaciones anuales & en resultados \\
- Las personas programan sus vacaciones & - Libertad en el horario \\
- No revisamos a la entrada o salida & \\
\hline
\end{tabular}

Como un paso previo a la categorización y apoyados en Bonilla y Rodríguez (2005), se construyeron redes conceptuales a partir de los distintos factores que emergían en el análisis de la información. Se trató de encontrar elementos y vínculos entre elementos que dieran cuenta de la toma de posiciones en los (nuevos) rasgos culturales.

Así entonces, se dirá que una vez se realizó el trabajo de campo, el investigador se dio a la tarea de construir las categorías de análisis que le permitieran ordenar la información proveniente de la encuesta, los grupos foco y entrevistas semiestructuradas, pues "la construcción de sentido a partir de los datos cualitativos implica un ejercicio de inmersión progresiva en la información, el cual comienza con un fraccionamiento del universo de análisis en subconjuntos de datos ordenados por temas, para luego recomponerlo en categorías" (Bonilla y Rodríguez, 2005, p. 251).

La interpretación se entiende como el proceso de análisis mediante el cual el investigador reconstruye uno de los posibles sentidos de la narración, aunque, por definición, puede presentar descripciones alternativas. Además, acudiendo a la corresponsabilidad (Galeano, 2004), se contrastaron los datos con los propios sujetos investigados, analizando hasta qué punto estos se sentían representados en la presentación de los hallazgos preliminares. Este procedimiento contribuyó con la construcción de categorías emergentes que se enriquecieron con el sentido y significado que los informantes les dan a estas.

De este modo, se le dio un nombre a fragmentos de información que eran recurrentes en los discursos. Así, empezaron a surgir algunos aspectos que configuraban las categorías emergentes, que en algunos casos se referían a rasgos culturales y en otros a medidas implementadas. Veamos entonces algunos hallazgos.

\section{Algunos hallazgos}

Esta sección del documento se ha organizado en cuatro partes. En la primera se expone brevemente una descripción de la manera como se implementa- ron las medidas en la organización. En la segunda parte se hablará de la aprehensión de nuevos rasgos culturales y la participación en este proceso del habitus como conjunto de esquemas de percepción, apreciación y evaluación. En la tercera, se exponen las acciones que emprenden los trabajadores es decir, la toma de posiciones, de acuerdo con lo descrito atrás, los bienes sociales que se construyen en el colectivo y los intercambios simbólicos generados. En la cuarta parte se dibujan las tensiones rastreadas en esta organización y las agrupaciones que se fueron dando entre los trabajadores (que también dan cuenta de toma de posiciones). Las expresiones de los participantes en el estudio se han puesto entre comillas mientras que los rasgos culturales que emergen en la transformación de la cultura se encuentran en cursiva.

La organización tomada como terreno para el estudio cuenta con más de 800 empleados. Esta es una empresa con más de 35 años de experiencia en el desarrollo de proyectos para industrias como Oil and Gas, Petroquímica y Complejos Industriales. La empresa hace gerencia de proyectos que abarcan el ciclo de vida completo, desde los estudios de factibilidad hasta la puesta en marcha, para el sector de crudo y gas y para la industria en general.

Como se dijo en la introducción, a partir de 2005 la gerencia decidió transformar la cultura organizacional. De esta manera, se iniciaron algunas medidas para reforzar la filosofía que se quería: Calidad a tiempo. $Y$ estas medidas se extendieron por siete años. Específicamente, algunas de estas medidas consistieron en libertad en el horario de trabajo, cambios en la denominación de procesos y áreas, eliminación de la supervisión directa a los ingenieros y la implementación de guías como estándares operacionales. En la Tabla 1 se presentan algunas de las medidas y rasgos culturales que se deseaba promover en la organización.

Estas ideas y medidas fueron implementándose gradualmente año tras año, a partir del 2005, teniendo como foco la filosofía de la organización: calidad a tiempo. No obstante, las maneras de trabajar, de relacionarse y los valores y medidas propuestos, 
empezaban a crear impactos y tensiones entre las personas, y a crear atmósferas que presionan a los miembros a adaptarse a determinadas formas de ver las cosas (Sánchez, Tejero, Yurrebaso, Lanero, 2006, p.75). La posibilidad de participar y aportar ideas, la existencia de un programa de inducción en el que se socializan propuestas, la presencia de un equipo de acompañamiento y las nuevas rutinas organizacionales como el tema de horarios y de guías con estándares operacionales, fueron medidas implementadas gradualmente durante 7 años. Estas medidas generaban inquietudes y preguntas en los trabajadores. Un ejemplo de ello es que en las entrevistas los informantes indicaban que a partir del programa de inducción, donde escuchaban que en esta empresa se daba la oportunidad de participar, se preguntaban si eso era real o era simplemente un discurso, como lo habían escuchado en otras empresas. Y con el paso de los años, los participantes sostienen que uno de los rasgos culturales allí es el entorno de participación. Veamos entonces, el proceso de configuración de nuevos rasgos culturales en esta organización.

\subsection{Los habitus en la aprehensión de rasgos culturales}

Para Sainsaulieu (1997) hablar de aprendizaje cultural significa hablar de la inculcación de códigos culturales de la vida en la empresa. Se trata de aprendizajes culturales ligados con la experiencia de cada persona. Por esta razón, en este apartado se expone la aprehensión de nuevos rasgos culturales y el papel de las disposiciones de los trabajadores en esa aprehensión.

Después de varios años de iniciado el proceso, estos son algunos rasgos culturales de la organización: la empresa ofrece servicios con calidad a tiempo, las personas tienen la disposición de participar en las decisiones y procesos, poseen la disposición a comprometerse en los proyectos que se les encomiendan, tienen la disposición a desempeñarse con responsabilidad y la disposición para trabajar en equipo. Estas disposiciones se pudieron evidenciar en el discurso de los informantes (durante las entrevistas y los grupos focales), en la descripción de la cultura de trabajo que hacen las personas y en la observación efectuada en la organización. Pero ¿cómo se configuraron esos y otros rasgos culturales? Aunque diversos factores pueden participar en ese proceso, este documento se ha focalizado en cómo las posiciones y la toma de posiciones de los trabajadores participan en la transformación de la cultura.

En la configuración de ciertos rasgos culturales como trabajo en equipo o autonomía, y en la aprehensión de valores corporativos, se encontraron, recurrentemente, situaciones en las que se aprecia una afinidad entre elementos de la gestión cultural y los habitus de las personas y lo que hace la gestión cultural es disparar o potenciar las disposiciones que los trabajadores traen producto de su historia personal. Es decir, cuando una manifestación cultural, como el valor corporativo respeto, hace parte de las disposiciones del empleado, esta manifestación le es, en algún sentido, más compatible. Esta compatibilidad o afinidad parece incidir, de acuerdo con las entrevistas y los grupos foco, en que el trabajador, a quien desde su casa le inculcaron el respeto, aprehenda este valor corporativo o rasgo cultural. Y esos habitus o disposiciones (Bourdieu, 2002) que el trabajador trae desde su casa, como el respeto, dan cuenta de posiciones del trabajador. Y recordemos una vez más, que se buscaba rastrear las posiciones de los trabajadores en la transformación de la cultura en esta organización. Esta situación se presentó, por lo general, en manifestaciones asociadas con valores, como por ejemplo la honestidad o el respeto.

De otro lado, a partir de la identificación de rasgos culturales que se asocian con las medidas implementadas, como la libertad en el horario o la autonomía para trabajar, se preguntó a los participantes si para ellos fue fácil o difícil aprehender o adaptarse a esas formas de trabajar. Y en el análisis de la información, a través del discurso, de acuerdo con la metodología de Wodak y Meyer (2003) y de la configuración de redes conceptuales, de acuerdo con Alvesson \& Karreman (2011), se rastrearon aquellas posiciones en las que los trabajadores siempre hablan en primera persona y desde allí argumentan su adaptación. Por ejemplo, frente a la flexibilidad en el horario son bastante comunes respuestas del tipo: "yo me adapté fácilmente porque yo soy una persona responsable", "porque toda la vida he sabido manejar mi tiempo", "para mí ha sido difícil porque me cuesta manejar mi tiempo" - "porque requiero de un supervisor para trabajar". Es decir, los informantes argumentan que su adaptación se debe en gran parte a sus propios esquemas mentales (Biggart y Beamish, 2003), conformados durante su historia personal. Ante la pregunta en el grupo foco ¿por qué ha sido fácil para usted adaptarse a esta medida? Los informantes sostienen que, incluso antes de llegar a esta empresa, ellos han sido personas que se caracterizan por ser responsables o comprometidos. Lo que una vez más da cuenta de la presencia de las disposiciones de los trabajadores a la hora de adaptarse a medidas propuestas por la dirección de la organización, las cuales contribuyeron con la transformación de su cultura.

Además, en el estudio de la configuración de nuevos rasgos culturales, se pudo rastrear que en ese proceso entran en juego algunos elementos asociados con las posiciones y toma de posiciones de los trabajadores como el sentido y el control social. 
En ocasiones, los trabajadores le asignan sentido a las formas de trabajar o de relacionarse en función de los resultados que se obtienen (Fiss y Zajac, 2006). Ejemplo de ello se vio al revisar pendientes o hacer el trabajo siguiendo unas guías con los estándares operacionales. En esta organización, encontrar sentido ayuda a la incorporación de ciertos hábitos en los trabajadores y de ciertos rasgos culturales.

De otro lado, en la empresa se ha creado un contexto de control social para el mantenimiento de ciertas prácticas de trabajo como la libertad en el horario o el seguimiento de estándares operacionales. Esta situación sugiere la presencia de un orden negociado entre actores, como lo propone Hallett (2003) al momento de analizar la configuración de la cultura. En el caso de la libertad de horario, los participantes afirman que es una situación en la que hay personas que abusan de esta posibilidad 4 . Sin embargo, ellos mismos se encargan de decirle al incumplido que es parte del respeto hacia los otros llegar puntual y que además de la libertad en el horario, la filosofía de la empresa proclama el cumplimiento con el clientes. Esta situación particular emergió recurrentemente en los grupos focales y se pudo también rastrear en las visitas de observación en la empresa. En síntesis, esta situación parece dar cuenta del sentimiento de control que han rastreado Bordia et al. (2004) en el contexto organizacional.

Estos elementos (sentido y control social) constituyen distintas fuerzas que entran en juego, simultáneamente, en ese espacio social. Fuerzas que están ligadas con las posiciones de los trabajadores y con la toma de posiciones de estos y que fueron objeto de búsqueda en el estudio. Emirbayer y Johnson (2008), plantean que en el espacio social existen distintos actores que están tomando posiciones no solo para ser aceptados sino para mantenerlas o mejorar esas posiciones. Un ejemplo de ello es el concurso del empleado del mes, o mejor aún, el empleado que mejor "encarna" la cultura de la organización. De acá se puede decir que las disposiciones de los empleados participan en la configuración de rasgos culturales porque potencian lo que se promueve en la organización y lo hacen a partir de los propios esquemas (de percepción y evaluación) de los trabajadores, como cuando estos le encuentran sentido a las medidas. Pero a su vez los otros trabajadores y sus propias disposiciones también llevan a configurar rasgos culturales a través del control social.

\subsection{La toma de posición a partir de la posición}

La configuración de rasgos culturales que se acaba de dibujar está avivada por dos aspectos particulares: las acciones que emprenden los trabajadores, como cuando deciden participar en decisiones y los intercambios simbólicos que se generan entre los trabajadores y la organización. A continuación se deslindan estos aspectos.

Como se dijo, Emirbayer y Johnson (2008) ven el campo organizacional desde dos ángulos: las posiciones objetivas de los actores y la toma de posiciones de esos actores. Para ellos, esto se corresponde con los conceptos de relaciones sociales y de cultura, respectivamente. Por consiguiente, estos autores sostienen que la organización puede ser abordada como un campo, en el que estas dos situaciones se constituyen mutuamente. En la empresa en estudio, los actores-trabajadores toman posiciones en los procesos de participación, buscando mantener su posición al hacer sentir sus opiniones y deseos al momento de participar. Los trabajadores adquieren o potencian la disposición a participar en las decisiones y toman posición cuando tienen una participación efectiva. Participan, ya sea porque le encuentran sentido, o porque se quieren integrar al grupo. Esta dinámica hace del trabajador un actor (Fernández, 2003), en la construcción de rasgos culturales, pues es él quien participa, quien toma posiciones.

Esa toma de posición y la condición particular de cada trabajador también pueden ser factores importantes a la hora de valorar bienes sociales presentes en la organización como la estabilidad laboral y la oportunidad de progresar profesionalmente ${ }^{6}$. Veamos un ejemplo. Al comparar las personas que tienen hijos con aquellas que no los tienen, en los primeros parece ser más generalizado el hecho de querer conservar la estabilidad laboral, mientras que en los segundos es más común el deseo de mantener la oportunidad de progresar profesionalmente ${ }^{7}$. Ahora bien, aquí solo se ha propuesto un ejemplo. Un ejemplo donde se pudo vincular (con las encuestas y los grupos foco) una condición particular de los trabajadores con un rasgo cultural específico que es reconocido y valorado por los trabajadores. Como este ejercicio, se hicieron otros que no se presentan acá por motivos de espacio. Pero lo que interesaba era analizar la toma de posición de cada trabajador, atada a su condición particular, al momento de valorar rasgos culturales que en este caso son bienes sociales. Ahora bien, son bienes sociales "en el sentido de que las personas saben que existen y además, que tienen derecho a hacer uso de ellos (Higuita y Grisales, 2014, p. 109). Esta situación sugiere que la posición objetiva que ocupa cada trabajador participa en la manera como se aprecian y valoran características del espacio social, que van configurando rasgos culturales. Y para analizar esto fue importante revisar, con la encuesta, variables sociodemográficas de los trabajadores como las que se aprecian en la Tabla 3. 


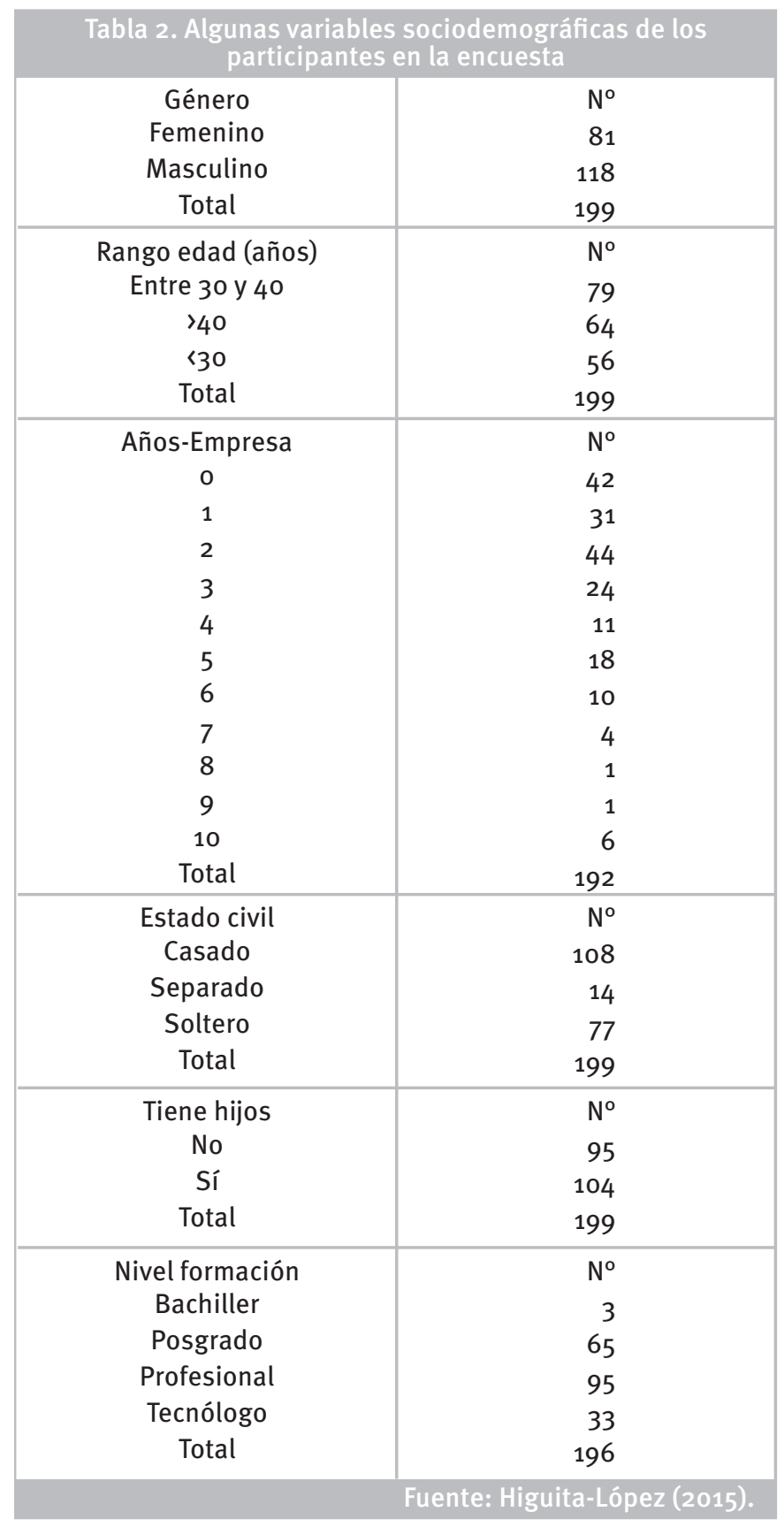

En términos de nivel jerárquico, en todo el proceso investigativo participaron 19 gerentes del nivel estratégico, 28 del nivel táctico y los demás eran ingenieros que hacen parte de los distintos proyectos de la empresa. Los trabajadores de esta organización son profesionales ( $90 \%$ ) y algunos tienen títulos de especialización o de maestría.Ahora bien, como lo que interesaba era rastrear las posiciones de los trabajadores y la toma de posiciones de estos, se puede decir que hay jefes, directivos y empleados que no están de acuerdo con políticas como la autonomía. Pero ¿cómo se han desenvuelto las fuerzas ante estas situaciones y tensiones en el campo? Los trabajadores, específicamente quienes se sienten muy a gusto con estas medidas, al ver que 1) sus compañeros trabajan con autonomía, 2) la gestión cultural la promueve y
Tabla 3. ¿Qué significa para usted trabajar en esta empresa?

\begin{tabular}{|c|c|c|c|c|}
\hline \multirow[t]{2}{*}{$\begin{array}{c}\text { ¿Qué significa } \\
\text { para usted } \\
\text { trabajar en } \\
\text { Energéticos? }\end{array}$} & \multicolumn{4}{|c|}{ Formación } \\
\hline & Bachiller & Posgrado & Profesional & Tecnólogo \\
\hline $\begin{array}{l}\text { Reconocimiento } \\
\text { por el trabajo }\end{array}$ & 1 & 18 & 43 & 16 \\
\hline $\begin{array}{l}\text { Ambiente de } \\
\text { confianza }\end{array}$ & 2 & 42 & 69 & 23 \\
\hline $\begin{array}{l}\text { Ser respetado } \\
\text { por compañeros } \\
\text { y directivos }\end{array}$ & 2 & 28 & 45 & 21 \\
\hline $\begin{array}{l}\text { Exigencia por la } \\
\text { calidad }\end{array}$ & 2 & 33 & 50 & 19 \\
\hline $\begin{array}{l}\text { Ser apoyado pro } \\
\text { sus colegas }\end{array}$ & 0 & 17 & 22 & 14 \\
\hline $\begin{array}{l}\text { Ser escuchado } \\
\text { por los jefes }\end{array}$ & 1 & 21 & 36 & 17 \\
\hline Un buen salario & 1 & 23 & 32 & 14 \\
\hline $\begin{array}{l}\text { Oportunidad de } \\
\text { participar en } \\
\text { decisiones }\end{array}$ & 1 & 12 & 27 & 17 \\
\hline $\begin{array}{l}\text { Satisfacción por } \\
\text { lo que se hace }\end{array}$ & 3 & 37 & 60 & 24 \\
\hline $\begin{array}{l}\text { Inconsistencia } \\
\text { entre la cultura } \\
\text { general y la } \\
\text { cultura de grupo }\end{array}$ & 0 & 6 & 6 & 3 \\
\hline $\begin{array}{l}\text { Oportunidad de } \\
\text { crecer personal- } \\
\text { mente }\end{array}$ & 3 & 36 & 51 & 23 \\
\hline $\begin{array}{l}\text { Flexibilidad para } \\
\text { trabajar }\end{array}$ & 3 & 43 & 72 & 24 \\
\hline $\begin{array}{l}\text { Presión por la } \\
\text { entrega a tiempo }\end{array}$ & 0 & 7 & 14 & 4 \\
\hline
\end{tabular}

3) la dirección los apoya, van entrando en esa dinámica de autonomía. Es decir, van asumiendo posiciones. Y muchos lo hacen a partir del capital social que han adquirido por su afinidad con las ideas de la gerencia. Pero ¿cómo opera allí el habitus?

El habitus produce una réplica cuyo principio no está inscrito en el estímulo y que, sin ser absolutamente imprevisible, no puede preverse a partir del mero conocimiento de la situación [...] una acción que, sin contradecirnos, podemos calificar 
a la vez de determinada y espontánea, porque está determinada por estimulaciones condicionales y convencionales que solo existen como tales para un agente dispuesto a percibirlas y apto para hacerlo (Bourdieu, 2002, p. 239).

En otras palabras, cuando los trabajadores sostienen (en los grupos focales y en las entrevistas) que "se van volviendo autónomos", se observa en su explicación un proceso de percepción y una toma de posición. Una percepción atada a la historia personal y una toma de posición atada a la posición objetiva que se ocupa en el espacio social. Y esto parece ser así, pues el habitus vincula las acciones a una trayectoria y a un campo a la vez, enterrados en el cuerpo, y producen una respuesta a la situación en el sentido práctico (Martínez, 2007, p. 317). Veamos un ejemplo.

La filosofía "calidad a tiempo" ha inspirado la idea de depositar confianza en los trabajadores. A partir de tal propósito se propicia, por parte de la gerencia, la autonomía de las personas. Como la dinámica de trabajo (con autonomía) se convierte en un hecho, las personas se ven abocadas a retribuir o compensar esa medida. Una manera de compensarla es, de acuerdo con las encuestas, entrevistas y grupos foco, actuando con responsabilidad y compromiso. Cuando los trabajadores reflexionan sobre la transformación cultural, manifiestan que han aprehendido (o potenciado) hábitos como ser responsable con los deberes y asumir el compromiso para hacer bien las cosas. Y ese aprendizaje está mediado por el contexto, como dirían Lam y Robertson (2010) y por las experiencias vividas con los otros en el lugar de trabajo (Sainsaulieu, 1997). En las entrevistas y en los grupos foco, los trabajadores expresan igualmente: "la empresa nos da, nosotros le damos". Es como un intercambio social, como si existiera un vínculo con el otro, que en este caso es la organización. Y asumir compromiso, que es una toma de posición, podría reforzar el rasgo cultural confianza (propuesto por la gerencia). Si nos apoyamos en Hallet (2003), este sostiene que la cultura emerge en la interacción, pero producto de un orden negociado en un espacio social, es decir, donde existen distintas fuerzas y diferentes posiciones. Sin embargo, se podría decir que estas fuerzas no son ecuánimes, pues para Hallet (2003) en ese orden negociado prevalecen aquellos que tienen mayor poder simbólico.

Precisamente Bourdieu (2002), estudia la dimensión simbólica del poder, para ver cómo visiones del mundo establecidas por grupos específicos se quieren imponer por sobre las de otros. Es importante mencionar que estas visiones del mundo o disposiciones de percepción y acción, se internalizan casi automáticamente, produciéndose con ello un proceso de "naturalización" (Hallet, 2003). Con esta concepción generalizada respecto a la percepción del mundo social se hace fácil ejercer control por parte del grupo dominante en el campo, porque ante cualquier intento por desviarse solo les basta apelar al sentido común (ya internalizado y aprehendido por los demás) para neutralizarlos y legitimar su dominación. Tal vez esta situación explique expresiones como la mencionada atrás, cuando se hablaba del intercambio social: "la empresa nos da [confianza], nosotros le damos [responsabilidad]".

Ahora bien, es claro que esas expresiones no tienen el mismo significado para todos los trabajadores y que este significado dependerá también de las condiciones y posiciones materiales de cada individuo en ese espacio social. De allí la importancia de revisar variables sociodemográficas. Tomemos otro ejemplo. Una de las preguntas de la encuesta fue: ¿qué significa para usted trabajar en esta empresa? Aunque entre los profesionales y los especialistas está más presente el ambiente que la satisfacción, entre los bachilleres y tecnólogos es esta última la más generalizada (Tabla 3 ).

El propósito de la Tabla 3 es mostrar que no todos los participantes valoran de la misma forma ciertos rasgos (que aparecen en las columnas) y que la cultura no es homogénea. Además, sabemos que se buscaba rastrear las posiciones y la toma de posiciones en la transformación cultural, por consiguiente, y con los ejemplos expuestos en este apartado, se puede decir que las acciones (y visiones) de los trabajadores son muestra de que ellos toman posiciones. Son ejemplos concretos las acciones como decidir participar o asumir el rol de ser autónomos. También, se puede decir que ese intercambio entre el trabajador y la organización está atravesado por las posiciones de aquel. Pero esas diversas visiones del espacio social, atadas a las posiciones, generan tensiones, como se describe a continuación.

\subsection{Las posiciones y las tensiones}

El conjunto de medidas implementadas en esta organización fueron generando dos tipos de tensiones. La primera derivada de la actitud de los trabajadores; la segunda, por el acuerdo o desacuerdo de estos frente a esas medidas. En este apartado se abordan las tensiones rastreadas en esta organización, el juego entre el individuo y el grupo mediante dinámicas de acople y de colectivización, y las agrupaciones que se fueron dando entre los trabajadores.

La primera tensión tiene su origen en la actitud que los trabajadores toman frente a las medidas propuestas. De acuerdo con los informantes, ante las propuestas de la Tabla 1, están los optimistas quienes lo ven como un "paraíso", los escépticos quienes están en relación directa con jefes que no están de acuerdo con medidas como participación de los trabajadores y los abusivos sobre todo en temas como la libertad en el horario o la flexibilidad para trabajar. Los optimistas son, principal pero no exclusivamente, jóvenes menores de 26 años, con menos de 3 años de antigüedad, ingenieros de distintas disciplinas. Su posición, al ver estas medidas 
como un "paraíso", ha sido participar activamente en su implementación con la intención de que se mantengan. Los escépticos son mayores de 40 años, ingenieros, que han laborado en otras organizaciones con estilos de liderazgo autocráticos. Su posición ha sido mantenerse al margen de la implementación de estas medidas porque están en relación directa con jefes que no están de acuerdo con ellas. Los abusivos pueden ser de cualquier edad o formación o nivel de antigüedad. Su posición ha sido apoyarse en medidas como la libertad en el horario para, por ejemplo, llegar tarde a reuniones de trabajo.

Pero fijemos algunas precisiones. Estas categorías (optimistas, etc.) fueron dadas por los mismos trabajadores en los grupos foco y en las entrevistas. No son categorías estrictamente delimitadas pues un mismo trabajador puede ser optimista frente a la libertad de horario y escéptico frente a la idea de la participación. Esta actitud (optimistas, etc.) y la consecuente toma de posición de los trabajadores presionan ciertas dinámicas en la formación de rasgos culturales, como se verá más adelante.

Estas tensiones empezaban a observarse desde el momento en que se hizo la prueba piloto de la encuesta. En esta prueba piloto, los mismos ingenieros sugirieron que se incluyera en el instrumento preguntas del tipo isu jefe o su grupo de trabajo vive la "cultura de calidad a tiempo"?, pues de acuerdo con ellos, hay algunos jefes que no aplican esa cultura.

El análisis de la información devela que las medidas mencionadas atrás apuntan a impactar formas de trabajar, maneras de relacionarse y modos de pensar en la organización. Sin embargo, en la medida en que los trabajadores se fueron involucrando y participando de este proceso, se observan, de acuerdo con Biggart y Beamish (2003), impactos en los modelos mentales de las personas.

La segunda tensión es generada por la convicción o no que se tenga frente a las medidas. Y esa convicción depende de las propias disposiciones de los trabajadores. Con el análisis de las encuestas (mediante cruces de variables a través de cubos olap) y del discurso en las entrevistas y los grupos focales, se rastrearon cuatro grupos de personas en torno a las nuevas ideas: 1) los promotores (el gerente, el equipo de psicólogas y algunos directivos), 2) los ingenieros y administradores que comulgan con estas ideas y que se autodenominaron multiplicadores, 3) los directivos y líderes de proyectos que no están de acuerdo con los cambios adelantados, y 4) un conjunto de trabajadores que "no" toma posición. El segundo grupo vive con la presión entre sentirse cómodos con medidas como la autonomía, la flexibilidad para participar, la libertad de horario y las acciones de unos directores de proyectos que no comulgan con esas ideas. "Sentirse cómodos" significa, de acuerdo con lo dicho párrafos atrás, que medidas como la autonomía o la libertad de horario han sido "naturalizadas", al menos para este conjunto de trabajadores. Este grupo trata de mantenerse firme (y esa es su manera de tomar posición) en su manera habitual de comportarse porque, de un lado, pueden y verbalizan esta presión en diferentes escenarios y del otro, saben que cuentan con el apoyo de la gerencia. Sobre esta situación, Martínez (2007, p. 258) sostiene que si los que están en posiciones inferiores dentro del campo necesitan de la acción colectiva es porque su desventaja en la posesión de recursos materiales y simbólicos hace mucho menos probable su inversión en este verdadero trabajo social que es la lucha por cambiar o perpetuar las reglas de juego en el campo. Pero ¿cómo se da esa lucha?

Los informantes mencionan que a través de los intercambios verbales con los otros, ellos "le van indicando a sus colegas esas maneras habituales de trabajar o de relacionarse". Y en estos intercambios verbales las personas se expresan y transmiten sus ideas, a través de la comunicación que un colectivo social comparte ideas y percepciones (Wodak y Meyer, 2003).

En esta dinámica las personas son constructoras de la realidad organizacional en la medida en que contribuyen a difundir o transmitir entre el colectivo (Kondra y Hurst, 2009) maneras aprendidas de proceder. Son actores en la medida en que participan en difundir, a través de los intercambios verbales, rasgos culturales. Expresiones como "tu puedes hacer así" o "usualmente lo hacemos así" dan cuenta de posiciones de los trabajadores.

$Y$ en este proceso el lenguaje ha sido fundamental. Expresiones como "es que aquí tú tienes autonomía" llevan al parecer a una construcción de ideas o creencias entre los trabajadores. Pero los trabajadores no son constructores de la realidad organizacional solamente porque transmiten en el colectivo rasgos culturales, sino porque en sí mismos no son entes inertes (Muñoz, 2010). En este sentido, Muñoz (2010, p. 251) plantea que en la configuración de cultura se da un "fenómeno de circularidad, pues la interacción humana en un contexto y una circunstancia específicos producen un sistema de representaciones que condicionará las interacciones en el futuro". Prueba de ello es que la posibilidad efectiva de participar que allí existe ha llevado a la conformación de un grupo de trabajadores que se ha denominado multiplicadores. Los trabajadores tomaron la posición de ser parte del grupo y además, como grupo, también toman posiciones. En estos grupos, los mismos trabajadores discuten sobre maneras de trabajar y sobre aspectos de la vida colectiva en términos de la cultura. Para ellos, estos grupos han sido muy importantes para el control social (del que ya se habló) y para "enseñarle a otros la cultura".

Los multiplicadores han establecido una cercanía con los gestores culturales, entre otras cosas, por su participación activa en las actividades que desde allí se impulsan. Y esa participación activa es una toma de posición. Ellos se encargan no solo de promover lo que la gestión cultural plantea, sino de ser ellos mismos promo- 
tores de nuevas iniciativas encaminadas, según ellos, a fortalecer y transmitir la "cultura", lo que expresa una clara toma de posición.

Por tanto, un elemento primordial fue observar las posiciones de los trabajadores frente a medidas como la libertad en el horario y los estándares operacionales. Para tal ubicación se tuvieron en cuenta el área, el nivel de formación, el rango de edad, el estado civil y si los trabajadores tenían o no hijos. Y como se dijo antes, en el análisis se buscó vincular condiciones particulares de los trabajadores con un rasgo cultural específico que es reconocido y valorado por ellos. Pero esto no significa que se pudieran rastrear y vincular todas las condiciones particulares de los trabajadores, ni que una condición esté vinculada solamente con un rasgo particular. A partir del análisis de las encuestas, se evidencian algunas diferencias, especialmente en lo referido al área de trabajo (Figura 1).

En términos generales, y de acuerdo con las encuestas, las variaciones frente a estándares operacionales no son tan marcadas como frente a la libertad en el horario, aspecto en que la adaptación ha sido más difícil para los trabajadores de calidad, control de documentos,pProceso, insumos y contratos.

Pero la transmisión de rasgos culturales no solo obedece al hecho de enseñar la manera de hacer las cosas. También a la tendencia de los sujetos a observar y a acoplarse con los otros (McDonough y Polzer, 2012). Y en este caso se encontraron dos situaciones (que se validaron con los mismos informantes en las entrevistas y en los grupos foco). La primera está asociada con la confrontación que hace un trabajador con su grupo inmediato. Es decir, las personas confirman que colegas y compañeros también han adquirido un determinado hábito. Por ejemplo, los trabajadores manifestaron que "los otros (sus compañeros) trabajan con autonomía, flexibilidad, confían en su equipo, o son responsables". Mediante el lenguaje y el comportamiento el individuo se acopla con el grupo, en un proceso social (Kondra y Hurst, 2009; Prue y Devine, 2012). En este proceso se van configurando rasgos culturales, en una dinámica concreta, porque los participantes sostienen "pues es que uno $v a$ aprendiendo de los otros y se va dando cuenta que sí se puede participar en decisiones". En otras palabras, los trabajadores se dan cuenta que sí se puede participar y toman la posición de participar.

La segunda situación está asociada con el hecho de adquirir ciertos hábitos (como el compromiso para hacer bien las cosas) lo que les da un lugar dentro de la compañía. A su vez, esto parece estar asociado con la idea de sentirse parte de. En esta dirección, los trabajadores afirman categóricamente: "es que trabajando con responsabilidad y compromiso, yo participo de este equipo que llamamos empresa. Yo ayudo a la construcción de este proyecto". Nótese allí la posición que cada uno ocupa y la idea de sentirse parte de. Es decir, haciendo su parte (aprendiendo a trabajar con responsabilidad) se hace parte de una empresa que trabaja con calidad y en la que existe confianza en los trabajadores. Aquí se revisa a Ignatow (2009) quien

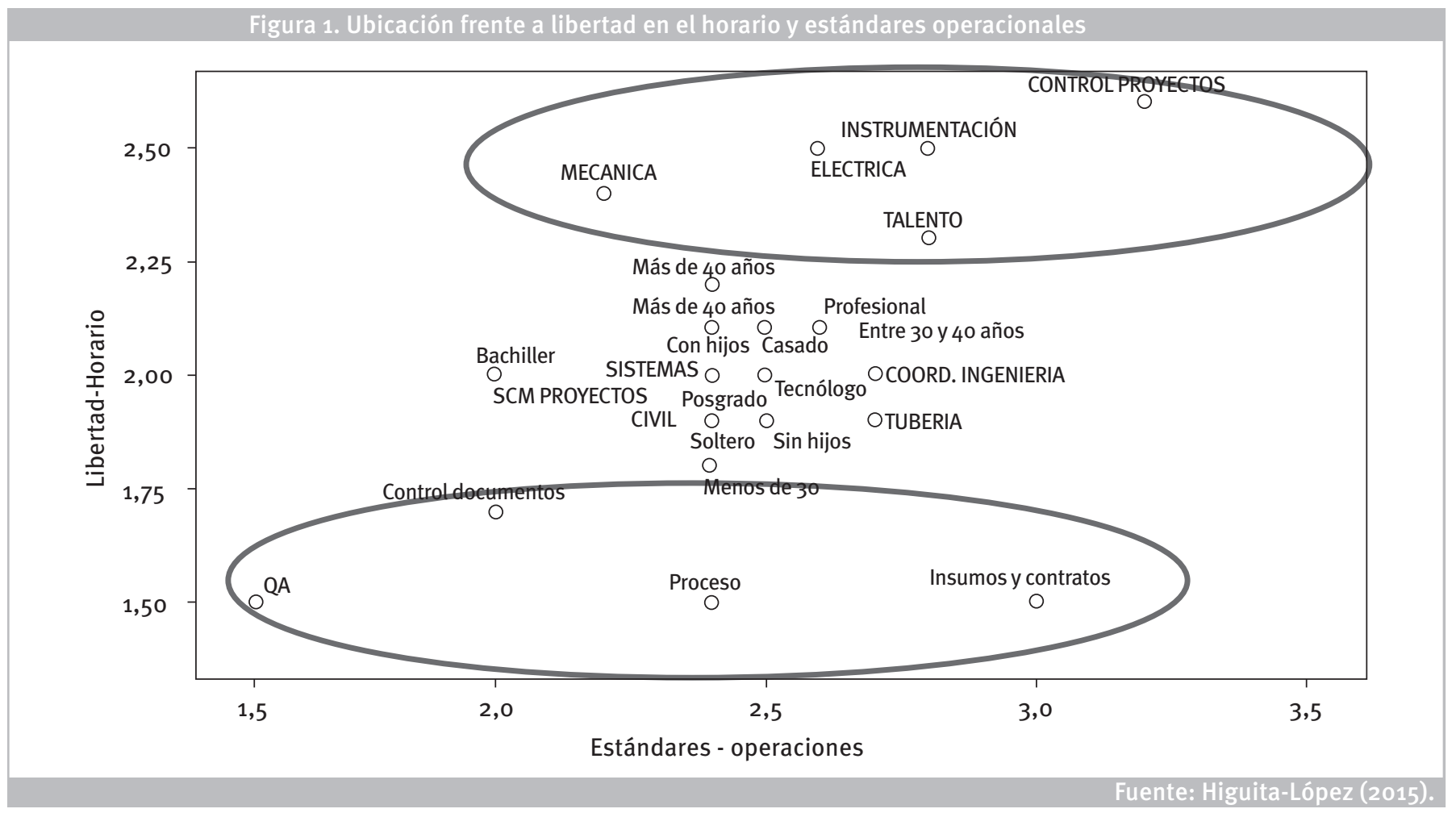


expresa que las metáforas del discurso y el habitus del individuo tienen un efecto en la adhesión social. Una metáfora de estas la encontramos en los trabajadores al referirse a la organización como "este proyecto" en el que se supone que "estamos todos involucrados". Aquí el individuo está tomando posiciones no solo para ser aceptado sino también para mantener sus posiciones o mejorar esas posiciones (Emirbayer y Johnson, 2008): haciendo su parte se hace parte de.

El análisis de encuestas también permitió ubicar los actores frente a rasgos culturales como el respeto - la oportunidad de crecer profesionalmente. Esta ubicación se hizo, como se aprecia en la Figura 2, considerando condiciones específicas de los trabajadores tales como el género, el estado civil, el nivel de formación, la antigüedad en la organización y la sede donde laboran. Pero se insiste. Aquí se muestran algunos de los análisis que se hicieron en los que se vinculaban posiciones de los trabajadores (y toma de posiciones) con la emergencia de nuevos rasgos culturales en la organización, asociados con aspectos referidos en la Tabla 1.

La Figura 2 permite decir que los trabajadores que más "valoran" bienes sociales como la oportunidad de crecer profesionalmente y el ambiente de respeto son hombres, casados, profesionales, con pocos años en la empresa y que laboran en la sede Serranías. Así entonces, la transformación cultural parece ser un proceso en el que cuenta la posición de los trabajadores y su toma de posición. Y ¿cómo toman posición? Pues toman posición, como se ha ejemplificado, cuando quieren compenetrarse con un grupo, cuando deciden participar, cuando asumen la autonomía como una forma de trabajo o cuando presionan a otros para asumir ciertas posiciones, como en las expresiones: "Es que esta es una empresa de ingeniería y no de embutidos". "Es que nosotros sí estamos apoyando el crecimiento de la compañía". Ya lo decía Martínez, "el habitus vincula las acciones a una trayectoria y a un campo a la vez, y producen una respuesta a la situación en el sentido práctico" (2007, p. 317).

Veamos un ejemplo más. Los trabajadores ingenieros le dan más importancia a valores como la responsabilidad y a hábitos como el compromiso, al compararlos con los trabajadores de la parte administrativa. En la Figura 3 se observa, por ejemplo, que las personas de sistemas, control de documentos, financiera y talento le otorgan a la responsabilidad y al compromiso calificaciones menores, lo que sugiere de nuevo que la valoración de los rasgos culturales dependen de las posiciones que, a su vez, avivan las tensiones descritas atrás.

El campo de fuerzas es un campo de luchas por transformar las relaciones de fuerzas, pero el modo como esta última se lleva a cabo supone el trabajo político (Sieweke, 2014). "Para Bourdieu, este trabajo

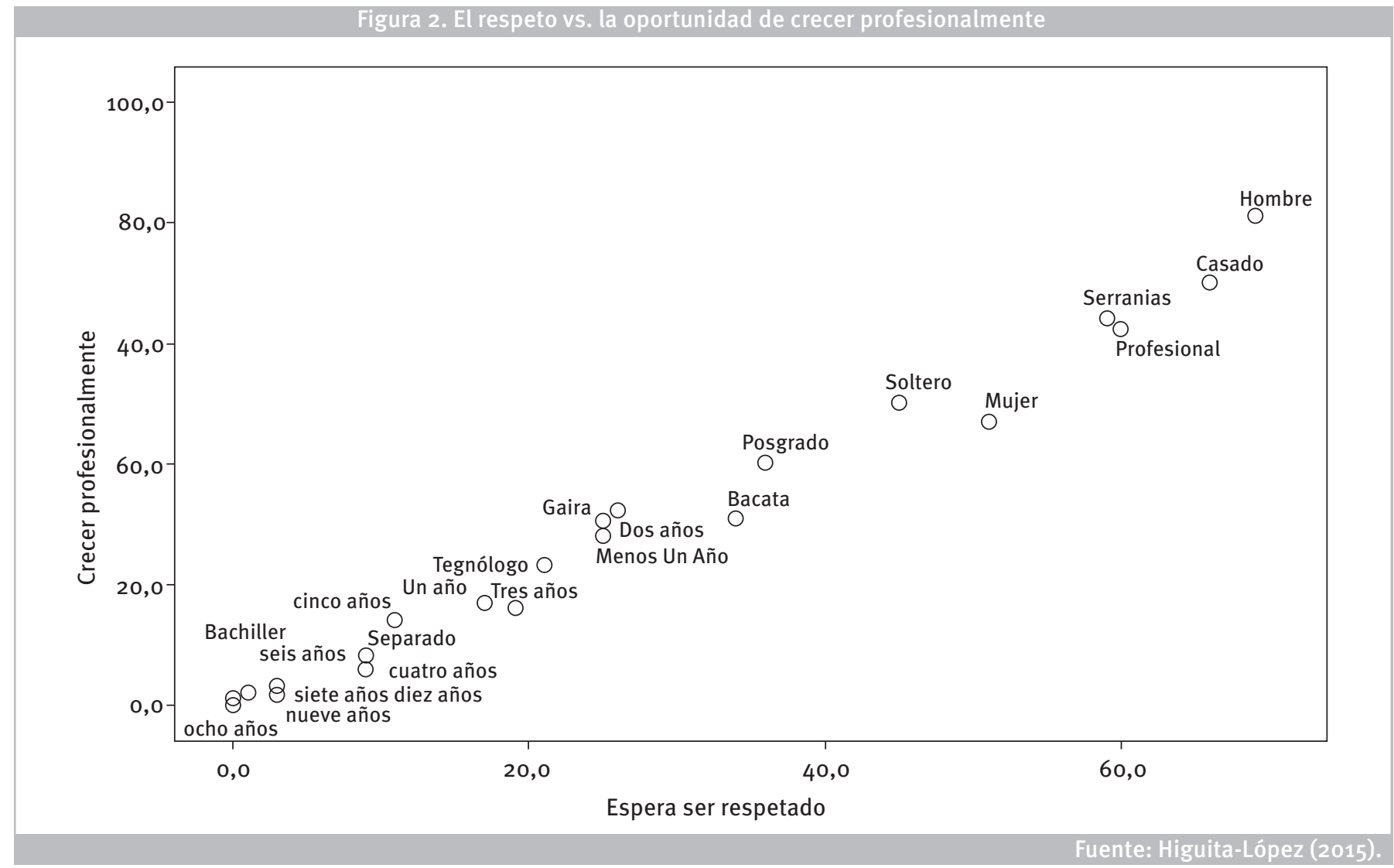


político es siempre acción simbólica, vinculada con el trabajo social de construcción de creencias: se trata de la imposición en el campo de un punto de vista, de un principio de visión y de división del espacio social" (Martínez, 2007, p. 257).

Ahora bien, surge la siguiente pregunta: ¿cómo han respondido los trabajadores que tienen jefes que no están de acuerdo con las medidas adoptadas? Pues son diferentes las posiciones que han tomado. Los más tímidos, se mantienen cautos, es decir, no hacen alarde de que en la empresa existe flexibilidad, autonomía y demás cosas. Ellos esperan allí, hasta que en un nuevo proyecto tengan la oportunidad de trabajar con otro director que sí comulgue con esa filosofía. Esperar es una forma de tomar posición. Otros han hecho parte del grupo de multiplicadores como una estrategia de generar presión sobre esos directivos que, de acuerdo con las palabras de los trabajadores en los grupos foco, "no se integran". Y hacer parte del grupo de multiplicadores es tomar posición. En estos grupos socializan sus inconformismos y las experiencias que han tenido y logran que en algunos casos, se tomen posiciones desde el área para mostrar que son pocos los que no comparten la filosofía de la organización o las medidas implementadas. Una de esas posiciones ha sido el concurso de cuál es el empleado que mejor representa la cultura de la organización. Un tercer grupo ha tenido en oportunidades, la ocasión de confrontar a esos directivos porque saben que tienen el apoyo de la gerencia. ¿Por qué lo tienen? Por la posición que ocupan en el espacio social, como lo sugieren Emirbayer y Johnson (2008), porque al estar más cerca de la gerencia, porque ocupan cargos administrativos, esto les provee de capital simbólico. Como consecuencia, estos trabajadores se apoyan en la gerencia para la confrontación, sin temor a ser despedidos. Ahora bien, es importante insistir que estas agrupaciones o clasificaciones que se hacen de los trabajadores, así como la toma de posiciones que aquí se han rastreado no son estáticas, pues un mismo trabajador puede estar de acuerdo con algunas medidas y en desacuerdo con otras.

Por todo lo anterior, el análisis llevó a agrupar a los actores/trabajadores de acuerdo con la toma de posiciones de estos. En grupos, esos actores son los gestores (gerente, equipo de psicólogas), los multiplicadores (ingenieros, personas de la parte administrativa y directivos de talento), opositores (directivos y líderes de proyectos que no están de acuerdo con las medidas y que, contrario a la gerencia, no creen que la cultura sea un factor importante en el desempeño organizacional) y el resto de trabajadores (que pueden

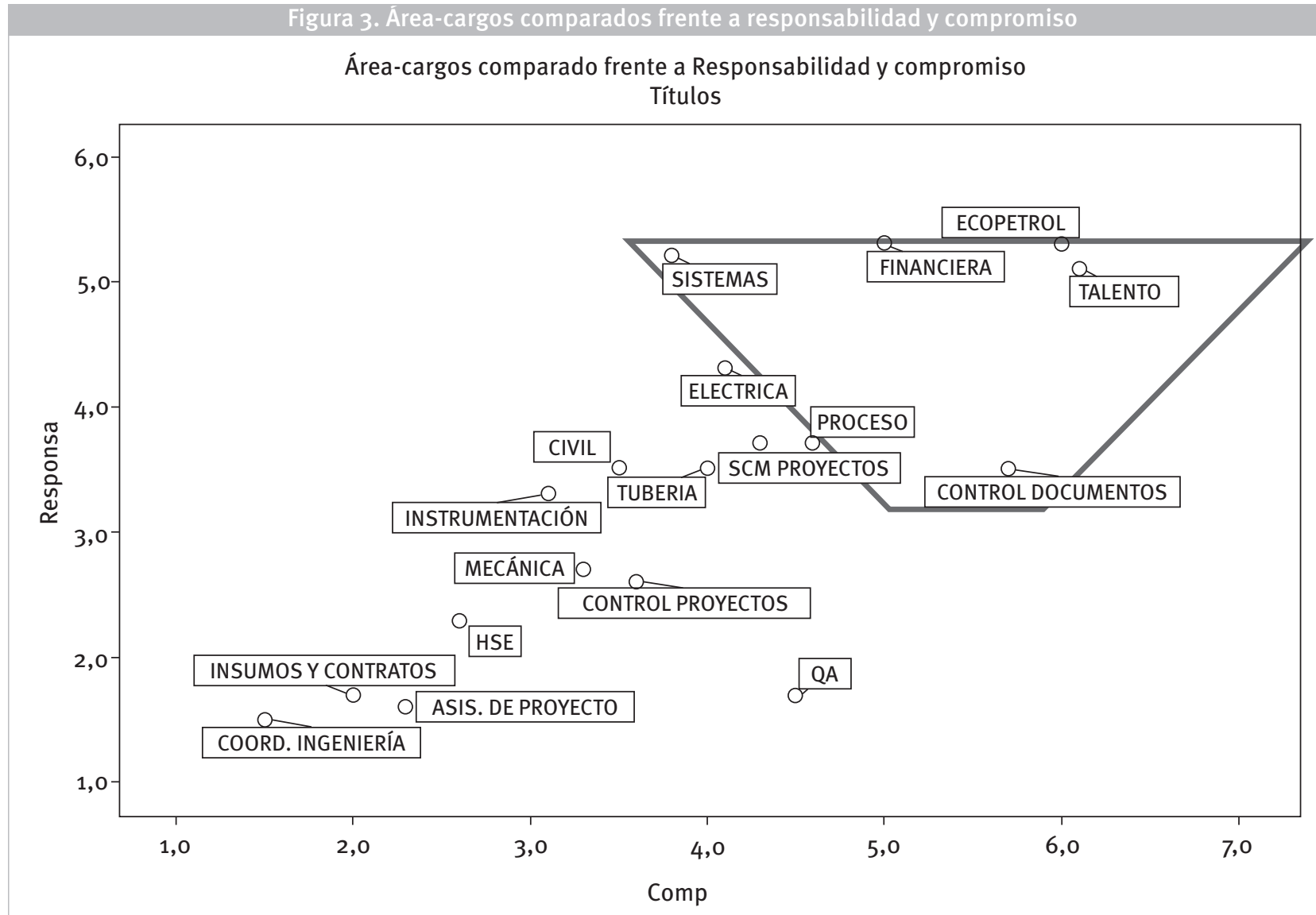


pertenecer tanto a ingeniería como a la parte administrativa). Los actores y las fuerzas presentes en el espacio social se exponen en la Figura 4.

¿Quiénes son los multiplicadores y quiénes los opositores presentes en este espacio social? Los multiplicadores son tanto hombres y mujeres entre 26 y 36 años. Tienen fundamental, pero no exclusivamente, menos de cinco años en la empresa, pertenecen a todas las sedes, son bachilleres, tecnólogos y profesionales. Los opositores, por su parte, tienen entre 35 y 45 años, son tanto mujeres como hombres, aunque más hombres que mujeres, por lo general, llevan más de ocho años en la compañía, pertenecen a todas las sedes, son profesionales y con grado de especialización.

Las tensiones generadas entre estos actores llevan, como hemos visto, a que las personas emprendan acciones y tomen posiciones y en esa medida se van configurando nuevas prácticas (formas de trabajar o de relacionarse) que se constituyen en rasgos culturales en esta organización. Además, las acciones de los trabajadores para acoplarse o para que otros aprehendan ciertos hábitos o comportamientos, evidencian que ellos toman posiciones.

\section{Conclusiones}

Es importante terminar diciendo que los análisis que aquí se han hecho sólo son válidos para la organización en estudio, y que este no tenía la pretensión de generalizar los hallazgos. Después de 7 años de iniciadas unas medidas en esta organización se pueden rastrear algunos rasgos culturales que dan cuenta de la transformación de su cultura. Adicionalmente, la toma de posiciones de los trabajadores ha sido uno de los factores clave (pero no el único) en esta transformación.

Por consiguiente, se puede decir que la transformación cultural también obedece a una acción colectiva, en la que participa la interacción social, los intercambios verbales y en la que el trabajador, más que un agente, es un actor. Es un trabajador que participa y propone, que se inquieta, se identifica y sobre todo se siente constructor de esa cultura cuando toma posición y declara: "la cultura la hacemos nosotros, la vivimos nosotros y la hacemos entre todos. Somos nosotros con la responsabilidad y el compromiso quienes hacemos de la empresa lo que es". Es decir, la identificación con rasgos culturales, como propone Watts (2010) también está presente en la transformación de la cultura de esta organización.

Por otro lado, las disposiciones de los empleados participan en la configuración de rasgos culturales porque potencian lo que se promueve en la organización. Son actores que toman posiciones y cuando lo hacen están participando en su propio proceso de modificación de sus disposiciones, lo que permite pensar que, efectivamente,

\section{Figura 4. Actores presentes en el espacio social}

Ambiente de trabajo

Compenetración con el grupo

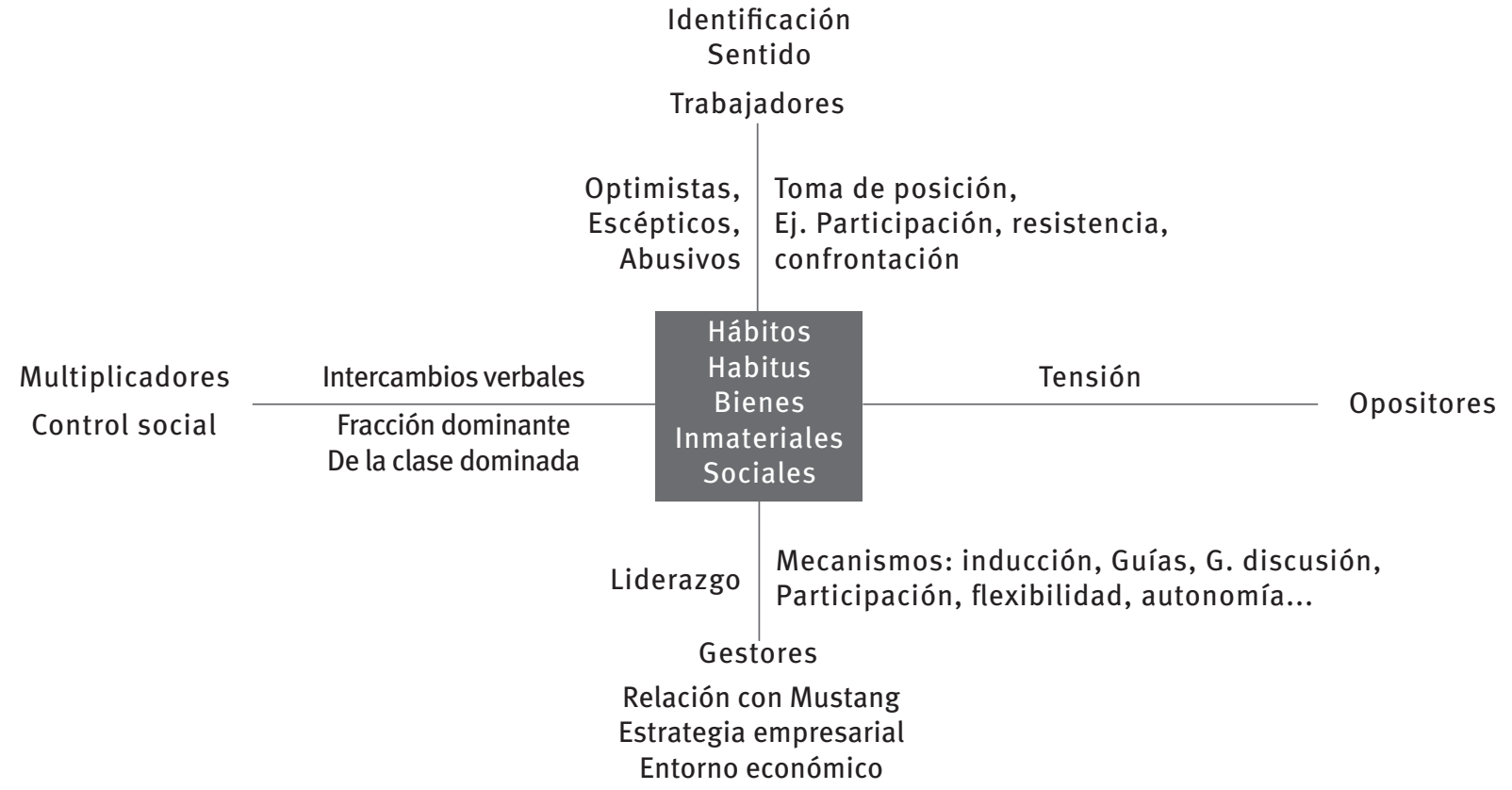

Mundo Material

Contratación a término indefinido, salarios competitivos, programas de capacitación técnica, plan de beneficios sociales extralegales, la posibilidad que tienen los empleados de trabajar en otras áreas, la posibilidad de diseñar sus propios espacios de trabajo, participar en procesos de selección de compañeros, etc. 
los trabajadores no son solo agentes que reproducen sus disposiciones en el campo. Se dirá, entonces con Fernández (2003), que más que agentes, los trabajadores son actores. Pero a su vez, los otros trabajadores y sus propias disposiciones, también llevan a configurar rasgos culturales a través del control social.

Las acciones de los trabajadores son muestra de que ellos toman posiciones. Acciones como decidir participar o asumir el rol de ser autónomos son claros ejemplos de ello. Y en algunos casos, esas acciones están asociadas con el intercambio social entre el trabajador y la organización. De igual forma, las tensiones generadas entre actores llevan a que las personas tomen posiciones, lo que contribuye con nuevas prácticas (formas de trabajar o de relacionarse) que se constituyen en rasgos culturales en esta organización.

Se terminará diciendo que la actuación de los trabajadores está ligada con la historia personal de cada uno, a sus habitus y la toma de sus posiciones. Y esa toma de posición está, a su vez, ligada con las posiciones de los trabajadores/actores en el espacio social que aquí se ha estudiado. En otras palabras, las condiciones materiales de cada trabajador y sus rasgos específicos hacen que valoren, evalúen y aprecien tanto los rasgos culturales como las condiciones de trabajo en la organización.

\section{Conflicto de interés}

Los autores declaran no tener conflicto de interés.

\section{Referencias}

Aktouf, O. (2002). El simbolismo y la cultura organizacional. De los abusos conceptuales a las lecciones de campo. $A D$ minister, 1(1), 63-93.

Alvesson, M. (1994). Talking in Organizations: Managing Identity and Impressions in an Advertising Agency. In, R.- Holt y F. den Hond (Eds.) Organization Studies (pp. 535-563). London, UK: SAGE.

Alvesson, M., \& Sveningsson, S. (2008). Changing organizational culture. Cultural change work in progress. London, Uk: Routledge.

Alvesson, M., \& Karreman, D. (2011). Qualitative research and theory development. London, UK: SAGE.

Biggart, N., \& Beamish, T. D. (2003). The economic sociology of Conventions: Habit, Custom, Practice, and Routine in Market Order. Annual Review of Sociology, 29(1), 443464.

Bonilla C., E., y Rodríguez., P. (2005). Más allá del dilema de los métodos. Bogotá, Colombia: Norma.

Bordia, P., Hobman, E., Jones, E., Gallois, C., \& Callan, V. J. (2004). Uncertainty during Organizational Change: Types, Consequences, and Management Strategies. Journal of Business \& Psychology, 18(4), 507-532.

Bourdieu, P. (2000). La distinción: criterios y bases sociales del gusto. Madrid, España: Taurus.

Bourdieu, P. (2002). Las estructuras sociales de la economía. Buenos Aires, Argentina: Manantial.
Bourdieu, P. (2007). Razones y lecciones de una práctica sociológica. Buenos Aires, Argentina: Manantial.

Bourdieu, P., y Wacquant, L. (1995). Respuestas, por una antropología reflexiva. México DF., México: Grijalbo.

Dalton, B. (2004). Creativity, habit, and the social products of creative action: revising jobs, incorporating bourdieu. Sociological Theory, 22(4), 603-622.

Ember, C., y Ember, M. (1997). El concepto de cultura. En Antropología cultural. Madrid, España: Prentice Hall.

Emirbayer, M., \& Johnson, V. (2008). Bourdieu and organizational analysis. Theory Sociology, 37(1), 1-44.

Fernández, 0. (2003). Pierre Bourdieu: ¿agente o actor? Tópicos del Humanismo. Recuperado 21/11/2006 http://www. geiuma-oax.net/Web/docs/agenteactor.htm

Fiss, P. C., \& Zajac, E. J. (2006). The Symbolic Management of Strategic Change: Sensegiving Via Framing and Decoupling. Academy of Management Journal, 49(6), 1173-1193.

Galeano, M. (2004). Estrategias de investigación social cualitativa: el giro en la mirada. Medellín, Colombia: La Carreta.

Gartman, D. (2007). The Strength of Weak Programs in Cultural Sociology: A Critique of Alexander's Critique of Bourdieu. Theory and Society, 36(5), 381-413.

Hallett, T. (2003). Symbolic power and organizational culture. Sociological Theory, 21(2), 128-149.

Higuita, D., y Grisales, L. (2014). La construcción de cultura en la organización: una mirada desde las representaciones colectivas en Lionel Vallée. Cuadernos de Administración, 27(48), 97-118.

Hofstede, G. (1999). Culturas y organizaciones. El software mental, la cooperación internacional y su importancia para la supervivencia. Madrid, España: Alianza Editorial.

Ignatow, G. (2009). Culture and Embodied Cognition: Moral Discourses in Internet Support Groups for Overeaters. Social Forces, 88(2), 643-689.

Lam, M., \& Robertson, D. (2010).The joint effect of organizational culture and tenure on employee attitude towards change: some preliminary data and findings. Proceedings of the Academy of Organizational Culture, Communications and Conflict, 15(1), 131-145.

Kondra, A. Z., \& Hurst, D. C. (2009). Institutional processes of organizational culture. Culture \& Organization, 15(1), 39-58.

Mangi, L. (2009). Neoinstitutionalism and the Appropriation of Bourdieu's Work: A Critical Assessment. Revista De Administração de Empresas, 49(3), 323-336.

Martínez, A. T. (2007). Pierre Bourdieu: razones y lecciones de una práctica sociológica. Buenos Aires, Argentina: Manantial.

McDonough, P., \& Polzer, J. (2012). Habitus, Hysteresis, and Organizational Change in the Public Sector. Canadian Journal of Sociology. 37(4) 357-379.

Muñoz, R. (2010). Cambio y cultura organizacionales. Una crítica teórica y un estudio de caso. En S. Arcand, R. Muñoz, J. Facal y J. Dupuis (Edi.), Sociología de la Empresa. Del marco histórico a las dinámicas internas (pp. 239-261). Bogotá, Colombia: Siglo del Hombre Editores.

Ojeda, H. (2009). Cultura organizacional y hábitus: análisis de un relato etnográfico. Investigación y Reflexión en Ciencias Sociales, (5), 45-68.

Prue, D., \& Devine, K. (2012). Isomorphism and organizational culture. A first Nation's housing initiative. International Journal of Indigenous Peoples, 8(3),253-263.

Sainsaulieu, R. (1997). Sociologie de l'entreprise, organisation, culture et développement. Paris, Francia: Presses de Sciences Politiques et Dalloz. 
Sánchez, J. C., Tejero, B., Yurrebaso, A., y Lanero, A. (2006). Cultura organizacional: desentrañando vericuetos. Revista de Antropología Iberoamericana, 1(3), 380-403.

Sieweke, J. (2014). Pierre Bourdieu in management and organization studies -A citation context analysis and discussion of contributions. Scandinavian Journal of Management, 30(4), 532-543.

Smircich, L. (1983). Concepts of Culture and Organizational Analysis. Administrative Science Quarterly, 28(3), 339-358.

Strategor. (1995) Estrategia, estructura, decisión, identidad. Política general de empresa. Barcelona, España: Biblioempresa.
Thevenet, M. (2013). La culture d'entreprise. Paris, France: PUF, 2010.

Watts, S. (2010). Identification and the Cultural Organization: How the Concept of Identification Can Impact the Constituencies Who Work Within Cultural Organizations. Journal of Arts Management, Law \& Society, 40(3), 200-216.

Wodak, R., y Meyer, M. (2003). Métodos de análisis crítico del discurso. Barcelona,España: Gedisa.

Zapata, A., y Rodríguez, A. (2008). Gestión de la cultura organizacional. Bases conceptuales para su implementación. Cali, Colombia: Universidad del Valle.

Cuadernos de Administración / Facultad de Ciencias de la Administración / Universidad del Valle Periodicidad: semestral / ISSN impreso N 0120-4645-ISSN electrónico N²256-5078 / Nombre abreviado: cuad.adm. Edición Vol. $31 \mathrm{~N}^{\circ} 53$ (enero - junio de 2015)

Acercamiento a un estudio sobre toma de posiciones y disposiciones en la organización. El caso de una empresa desde la perspectiva Bourdiana / Daimer Higuita López 